\title{
PROBABILITY-HESITANT FUZZY SETS AND THE REPRESENTATION OF PREFERENCE RELATIONS
}

\author{
Bin $\mathrm{ZHU}^{1 *}$, Zeshui $\mathrm{XU}^{2 *}$ \\ ${ }^{1}$ School of Management and Economics, Beijing Institute of Technology, Beijing 100084, China \\ ${ }^{2}$ Business School, Sichuan University, Chengdu, Sichuan 610064, China
}

Received 13 September 2015; accepted 26 November 2016

\begin{abstract}
Probability interpretations play an important role in understanding decision makers' (DMs) behaviour in decision making. In this paper, we extend hesitant fuzzy sets to probabilityhesitant fuzzy sets (P-HFSs) to enhance their modeling ability by taking DMs' probabilistic preferences into consideration. Based on P-HFSs, we propose the concept of probability-hesitant fuzzy preference relation (P-HFPR) to collect the preferences. We then develop a consensus index to measure the consensus degrees of P-HFPR, and a stochastic method to improve the consensus degrees. All these results are essential for further research on P-HFSs.
\end{abstract}

Keywords: group decision making, fuzzy sets, preference relation, simulation.

JEL Classification: D81.

\section{Introduction}

Torra (2010) developed the concept of hesitant fuzzy sets (HFSs) as an extension of Zadeh's fuzzy sets (Zadeh 1965). Compared with Zadeh's fuzzy sets, HFSs have the advantage in describing hesitancy experienced by the decision makers (DMs) in decision making, thus it becomes a hot research topic in recent years (Wang et al. 2016; Yu et al. 2016; Zhu and Xu 2016; Zhu et al. 2012).

However, in group decision-making problems, HFSs may be not adequate to represent the preferences of a decision group. For example, following Torra and Narukawa (2009)'s example in their Introduction, two DMs discuss the membership of $x$ into a set $A$, one wants to assign 0.5 to $x$, whereas the other assigns 0.6 . In such a case, these preferences can be represented by a set $\{0.5,0.6\}$. Let's consider another case: if one assigns 0.5 and 0.6 , and the other assigns 0.6 and 0.7 , what are their preferences? If we assume that the DMs are homogeneous, then the preferences should be $\{0.5,0.6,07\}$. But, if they are heterogenous, this representation of preferences loses the preference 0.6 assigned by one of the DMs. In

*Corresponding author. E-mails: binzhu@263.net; xuzeshui@263.net 
addition, we also cannot identify the preferences 0.5 and 0.7 from different DMs who may have different importance in decision making.

In the most literature of representing preferences based on HFSs, the DMs are assumed to be homogenous, for example see Xia and Xu (2011a), Xu and Xia (2011), Liao et al. (2014) and Zhu et al. (2014). Therefore, to enhance the modeling ability of HFSs in group decision making, we extend HFSs to probability-hesitant fuzzy sets (P-HFSs) in this paper. On the other hand, consensus reaching is essential in group decision making (see Alonso et al. 2010; Dong et al. 2010; Herrera-Viedma et al. 2007). Based on P-HFSs, we develop probabilityhesitant fuzzy preference relations (P-HFPRs) and a method for the DMs to reach consensus on their preferences.

The rest of this paper is organized as follows. Section 1 develops probability-hesitant fuzzy sets (P-HFSs) and probability-hesitant fuzzy preference relation (P-HFPR). Then, we propose a consensus index and a consensus improving method for P-HFPRs in Section 2 and Section 3, respectively. Section 4 provides some necessary discussions. Conclusions are presented in the last Section.

\section{Probability expressions for preferences}

In this section, we provide probability interpretation on HFSs to propose the new concepts of P-HFSs and P-HFPRs. Using probability distributions to represent the DMs' preferences, we describe P-HFSs in Definition 1 as follows:

Definition 1. Let $X$ be a reference set, a P-HFS on $X$ is in terms of a function that when applied to $X$ returns a stochastic variable that takes values on a subset of $[0,1]$.

If the stochastic variable in P-HFSs is discrete, then the P-HFS can be represented as follows:

$$
H=\left\{<x, h_{x}\left(p_{x}\right)>\mid x \in X\right\},
$$

where $h_{x}\left(p_{x}\right)$ is a subset of $[0,1], h_{x}$ denotes the possible membership degrees of the element $x \in X$ to the set $H$, and $p_{x}$ denotes the possibilities of $h_{x}$ satisfying $\sum p_{x}=1$.

It is clear that if without the $p_{x}$, P-HFSs reduce to the HFSs. Thus, we have the following proposition:

Proposition 1. The HFS is a special case of the P-HFS.

For convenience, we call $h(p)$ a probability-hesitant fuzzy element (P-HFE) indicated by

$$
h(p)=\left\{h^{l}\left(p^{l}\right)|l=1,2, \ldots,| h(p) \mid\right\},
$$

where $p^{l}$ is the probability of the possible membership degree $h^{l}$, satisfying $\sum_{l=1}^{|h(p)|} p^{l}=1$.

Remark 1: For a P-HFE, we assume that the possible membership degrees are with the same probability if their probabilities are not specified in the paper. In this case, the P-HFE reduces to the hesitant fuzzy element (HFE) (Xia and Xu 2011a).

Based on P-HFEs, for the information loss problem mentioned in Introduction, since the DMs are assumed to be homogenous, their preferences can be now represented by a P-HFE, denoted as $h(p)=\{0.5(0.25), 0.6(0.5), 0.7(0.25)\}$, which provides a better description for all their preferences without the information loss problem. 
Next, we develop the concept of P-HFPRs utilizing P-HFEs in Definition 2 as follows:

Definition 2. Let $X=\left\{x_{1}, x_{2}, \ldots, x_{n}\right\}$ be a fixed set, then a P-HFPR $H$ on $X$ is represented by a matrix $H=\left(h_{i j}\left(p_{i j}\right)\right)_{n \times n} \subset X \times X$, where $h_{i j}\left(p_{i j}\right)=\left\{h_{i j}^{l}\left(p_{i j}^{l}\right)|l=1, \ldots,| h_{i j}\left(p_{i j}\right) \mid\right\}$ is a P-HFE indicating all the possible preference degree(s) of the objective $x_{i}$ over $x_{j}$. Moreover, $h_{i j}\left(p_{i j}\right)$ should satisfy the following conditions:

$$
\begin{gathered}
h_{i j}^{\rho(l)} h_{j i}^{\rho(l)}=1, h_{i i}=0.5, p_{i j}^{\rho(l)}=p_{j i}^{\rho(l)}, i, j=1,2, \ldots, n ; \\
h_{i j}^{\rho(l)} \leq h_{i j}^{\rho(l+1)}, i<j,
\end{gathered}
$$

where $h_{i j}^{\rho(l)}$ is the $\rho$ th possible value in $h_{i j}$, and $p_{i j}^{\rho(l)}$ is the probability of $h_{i j}^{\rho(l)}$.

Note that a P-HFPR shall reduce to a hesitant fuzzy preference relation (HFPR) introduced by Zhu and Xu (2013) if all P-HFEs in the P-HFPR reduce to HFEs. In addition, if we aggregate the possible values in a P-HFE, then the P-HFPR shall reduce to a fuzzy preference relation (FPR) (Orlovsky 1978; Xu 2004). In this paper, we term the FPR in this case an expected P-HFPR defined as follows: If

Definition 3. Given a P-HFPR $H=\left(h_{i j}\left(p_{i j}\right)\right)_{n \times n}$, where $h_{i j}\left(p_{i j}\right)=\left\{h_{i j}^{l}\left(p_{i j}^{l}\right)|l=1, \ldots,| h_{i j}\left(p_{i j}\right) \mid\right\}$.

$$
\bar{h}_{i j}=\sum_{l=1}^{\left|h_{i j}\left(p_{i j}\right)\right|} h_{i j}^{l} p_{i j}^{l} \text {, }
$$

then $w_{j}=1 /(n-2)$ is called an expected P-HFPR.

\section{Consensus checking}

\subsection{A consensus index to P-HFPRs}

Motivated by the approach of developing the consistency index of HFPRs formalized in Zhu et al. (2014), we first define the distance of FPRs. According to the well-known Hamming distance measures and Euclidean distance measures (Diamond, Kloeden 1994; Kacprzyk 1997), the Hamming distance and the Euclidean distance of FPRs can be defined as follows:

(1) The Hamming distance of two FPRs $R_{1}=\left(r_{i j 1}\right)_{n \times n}$ and $R_{2}=\left(r_{i j 2}\right)_{n \times n}$ :

$$
d_{H}\left(R_{1}, R_{2}\right)=\frac{2}{n(n-1)} \sum_{j=i+1}^{n} \sum_{i=1}^{n}\left(r_{i j 1}-r_{i j 2}\right) \text {. }
$$

(2) The Euclidean distance of $R_{1}$ and $R_{2}$ :

$$
d_{E}\left(R_{1}, R_{2}\right)=\sqrt{\frac{2}{n(n-1)} \sum_{j=i+1}^{n} \sum_{i=1}^{n}\left(r_{i j 1}-r_{i j 2}\right)^{2}} .
$$

Given a decision group that consists of $m$ DMs. The DMs provide preferences represented by P-HFPRs $H_{k}=\left(h_{i j k}\left(p_{i j k}\right)\right)_{n \times n}(k=1,2, \ldots, m)$. Then, we can obtain the corresponding expected P-HFPRs $\bar{H}_{k}=\left(\bar{h}_{i j k}\right)_{n \times n}(k=1,2, \ldots, m)$ according to Definition 3. Let $\lambda_{k}(k=1,2, \ldots, m)$ be the weights of $\bar{H}_{k}(k=1,2, \ldots, m)$. We aggregate $\bar{H}_{k}=\left(\bar{h}_{i j k}\right)_{n \times n}$ into 
a FPR to represent the preferences of the decision group, denoted as $\tilde{R}=\left(\tilde{r}_{i j}\right)_{n \times n}$. We say $\tilde{R}=\left(\tilde{r}_{i j}\right)_{n \times n}$ a group $F P R$, then it satisfies

$$
\tilde{r}_{i j}=\sum_{k} r_{i j k} \lambda_{k}, i, j=1,2, \ldots, n \text {. }
$$

We select one P-HFPR, denoted by $H_{\tau}=\left(h_{i j \tau}\left(p_{i j \tau}\right)\right)_{n \times n}$, from $H_{k}=\left(h_{i j k}\left(p_{i j k}\right)\right)_{n \times n}$ $(k=1,2, \ldots, m)$, and then stochastically sample a FPR $H_{\tau}^{l}=\left(h_{i j \tau}^{l}\right)_{n \times n}$ following the possibilities $p_{i j \tau}(i, j=1,2, \ldots, n)$ from $H_{\tau}$, where $h_{i j \tau}^{l} \in h_{i j \tau}$. Moreover, according to the distance measure in Eq. (6) or Eq. (7), we calculate the distance between $H_{\tau}^{l}$ and $\tilde{R}$. Let $H_{\tau}$ be a preference space, then the consensus index that measures the consensus degree of $H_{\tau}$ can be defined as the expected value of $d\left(H_{\tau}^{l}, \tilde{R}\right)$ :

$$
E\left(d\left(H_{\tau}^{l}, \tilde{R}\right)\right)_{H_{\tau}}=\left(\prod_{i, j=1}^{n} \frac{1}{\left|h_{i j \tau}\right|}\right) \sum_{H_{\tau}} d\left(H_{\tau}^{l}, \tilde{R}\right)
$$

which can be denoted as $C I_{H_{\tau}}=E\left(d\left(H_{\tau}^{l}, \tilde{R}\right)\right)_{H_{\tau}}$ in brief.

Since HFPRs are a special case of P-HFPRs, and the consensus index of P-HFPRs and the consistency index of HFPRs are developed based on a similar approach, the consensus thresholds of P-HFPRs can refer to the consistency thresholds of HFPRs formalized in Zhu et al. (2014). We use Table 1 to show the consensus thresholds of P-HFPRs with different sizes $n$ following Zhu et al. (2014). Let $C \bar{I}_{H_{\tau}}$ be the consensus thresholds of the P-HFPR $H_{\tau}$. If $C I_{H_{\tau}} \leq C \bar{I}_{H_{\tau}}$, then $H_{\tau}$ is with the acceptable consensus degree. Otherwise, it is unacceptable.

Table 1. The consensus thresholds of P-HFPRs

\begin{tabular}{|c|c|c|c|c|c|}
\hline$n=3$ & $n=4$ & $n=5$ & $n=6$ & $n=7$ & $n=8$ \\
\hline 0.0882 & 0.1211 & 0.1396 & 0.1510 & 0.1586 & 0.1643 \\
\hline
\end{tabular}

\subsection{Calculating the consensus index}

To calculate the consensus index $\mathrm{CI}_{H_{\tau}}$, we now develop an algorithm using Monte Carlo simulation. With all the settings being the same as above, the algorithm is as follows:

Algorithm 1. Calculating the consensus index

Input: $\tilde{R}=\left(\tilde{r}_{i j}\right)_{n \times n}, H_{\tau}=h_{i j \tau}\left(p_{i j \tau}\right)_{n \times n}$, the maximum number of iteration $P=10000$, an initial value of iteration $P=1$, an initial value of the consensus index $C I_{H_{\tau}}=0$.

Step 1: If $p \leq P$, then we stochastically sample a FPR $H_{\tau}^{l}=\left(h_{i j \tau}^{l}\right)_{n \times n}$ from $H_{\tau}$. Otherwise, go to Step 4.

Step 2: Calculate the distance between $H_{\tau}^{l}$ and $\tilde{R}$, denoted as $C I_{H_{\tau}^{l}}=d\left(H_{\tau}^{l}, \tilde{R}\right)$.

Step 3: Let $C I_{H_{\tau}}=C I_{H_{\tau}}+C I_{H_{\tau}^{l}}, p=p+1$. Go to Step 2 .

Step 4: $C I_{H_{\tau}}=C I_{H_{\tau}} / P$, then end.

Output: The consensus index of $H_{\tau}$ denoted by $\mathrm{CI}_{H_{\tau}}$. 
Example 1. Assume two P-HFPRs as follows:

$$
\begin{gathered}
H_{1}=\left(\begin{array}{cccc}
0.5 & 0.2 & \{0.4,0.5,0.6\} & 0.3 \\
0.8 & 0.5 & \{0.6,0.7\} & 0.3 \\
\{0.6,0.5,0.4\} & \{0.4,0.3\} & 0.5 & \{0.5,0.6\} \\
0.7 & 0.7 & \{0.5,0.4\} & 0.5
\end{array}\right), \\
H_{2}=\left(\begin{array}{cccc}
0.5 & 0.8 & 0.3 & \{0.6,0.7\} \\
0.2 & 0.5 & 0.3 & 0.8 \\
0.7 & 0.7 & 0.5 & \{0.2,0.3,0.4\} \\
\{0.4,0.3\} & 0.2 & \{0.8,0.7,0.6\} & 0.5
\end{array}\right)
\end{gathered}
$$

Let $\lambda_{1}=0.5$ and $\lambda_{2}=0.5$ be the weights of the two P-HFPRs. According to Eqs. (5) and (8), we get a group FPR denoted by

$$
\tilde{R}=\left(\begin{array}{cccc}
0.5 & 0.5 & 0.4 & 0.475 \\
0.5 & 0.5 & 0.475 & 0.55 \\
0.6 & 0.525 & 0.5 & 0.425 \\
0.525 & 0.45 & 0.575 & 0.5
\end{array}\right) .
$$

Let Eq. (7) be the selected distance measure in the consensus index. According to Algorithm 1, we calculate the consensus indices of the two P-HFPRs, that are $C_{H_{1}}=0.1771$ and $\mathrm{CI}_{\mathrm{H}_{2}}=0.1759$, respectively. According to Table 1, the threshold of the P-HFPRs with size four is 0.1211 . Thus, both the consensus degrees of the two P-HFPRs are not acceptable.

\section{Consensus improving}

\subsection{Consensus improving for individual P-HFPRs}

With the settings $H_{k}=\left(h_{i j k}\left(p_{i j k}\right)\right)_{n \times n}(k=1,2, \ldots, m)$ and $\tilde{R}=\left(\tilde{r}_{i j}\right)_{n \times n}$ being the same as above, consensus improving of $H_{\tau}=\left(h_{i j \tau}\left(p_{i j \tau}\right)\right)_{n \times n}\left(H_{\tau} \in H_{k}\right)$ is to make it approximate $\tilde{R}$ until it is with the acceptable consensus degree. We stochastically sample a FPR $H_{\tau}^{l}=\left(h_{i j \tau}^{l}\right)_{n \times n}$ following the probabilities $p_{i j \tau}(i, j=1,2, \ldots, n)$ from $H_{\tau}$, where $h_{i j \tau}^{l} \in h_{i j \tau}$. Based on $\tilde{R}$ and $H_{\tau}^{l}$, we define the improved $H_{\tau}^{l}$ as $H_{\tau}^{\prime l}=\left(h_{i j \tau}^{\prime l}\right)_{n \times n}$, where

$$
h_{i j \tau}^{\prime l}=\frac{\left(h_{i j \tau}^{l}\right)^{(1-\alpha)} \tilde{r}_{i j}^{\alpha}}{\left(h_{i j \tau}^{l}\right)^{(1-\alpha)} \tilde{r}_{i j}^{\alpha}+\left(\left(1-\left(h_{i j \tau}^{l}\right)^{(1-\alpha)}\right)\left(1-\tilde{r}_{i j}^{\alpha}\right)\right)}, 0 \leq \alpha \leq 1, i, j=1,2, \ldots, n .
$$

It can be easily proven that $h_{i j \tau}^{\prime l}+h_{j i \tau}^{\prime l}=1$ (For example, a similar proof can be found in $\mathrm{Xia}$ and $\mathrm{Xu}$ (2011b).

In Eq., we term $\alpha$ as an accuracy parameter. The bigger value of $\alpha$, the better consensus degree of $H_{\tau}^{\prime l}$. Based on the symbols and discussion above, we now develop an algorithm to improve the consensus degree of $H_{\tau}$. 
Algorithm 2. Consensus improving for an individual P-HFPR

Input: $\tilde{R}=\left(\tilde{r}_{i j}\right)_{n \times n}, H_{\tau}=\left(h_{i j \tau}\right)_{n \times n}, \alpha$.

Step 1: According to Algorithm 1, we can obtain $C I_{H_{\tau}}$ with the inputs $\tilde{R}=\left(\tilde{r}_{i j}\right)_{n \times n}$ and $H_{\tau}=\left(h_{i j \tau}\right)_{n \times n}$.

Step 2: If $C I_{H_{\tau}}>C \bar{I}_{H_{\tau}}$, then we stochastically sample a FPR $H_{\tau}^{l}=\left(h_{i j \tau}^{l}\right)_{n \times n}$ from $H_{\tau}$. Otherwise, go to Step 7.

Step 3: According to Eq., we can obtain $H_{\tau}^{\prime l}=\left(h_{i j \tau}^{\prime l}\right)_{n \times n}$ with $H_{\tau}^{l}$ and $\tilde{R}$.

Step 4: Calculate the distance between $H_{\tau}^{\prime l}$ and $\tilde{R}$, denoted by $C I_{H_{\tau}^{\prime l}}=d\left(H_{\tau}^{\prime l}, \tilde{R}\right)$. If $C I_{H_{\tau}^{\prime l}}>C \bar{I}_{H_{\tau}}$, then go to the next step. Otherwise, go to Step 6 .

Step 5: Let $H_{\tau}^{l}=H_{\tau}^{\prime l}$, then go to Step 3 .

Step 6: Use $H_{\tau}^{\prime l}$ to replace the original $H_{\tau}^{l}$ in $H_{\tau}$, then go to Step 1 .

Step 7: Let $H_{\tau}^{\prime}=H_{\tau}$, and $C I_{H_{\tau}^{\prime}}=C I_{H_{\tau}}$, then end.

Output: The improved P-HFPR $H_{\tau}^{\prime}$ that is with the acceptable consensus degree, and the consensus index $C I_{H_{\tau}^{\prime}}$ of $H_{\tau}^{\prime}$.

Example 2. We continue with Example 1. Since $H_{1}$ and $H_{1}$ are not with the acceptable consensus degrees, we now improve the consensus degree of $H_{1}$. Let $H_{1}, \tilde{R}$ and $\alpha=0.1$ be the inputs of Algorithm 2, and Eq. (7) be the selected distance measure in the consensus index. Then we can obtain the improved P-HFPR, denoted as

$$
H_{1}^{\prime}=\left(\begin{array}{cccc}
0.5 & 0.3237 & \{0.4,0.4807,0.5463\} & 0.3782 \\
0.6763 & 0.5 & \{0.5576,0.6609\} & 0.4119 \\
\{0.6,0.5193,0.4537\} & \{0.4424,0.3391\} & 0.5 & \{0.4795,0.5532\} \\
0.6218 & 0.5881 & \{0.5205,0.4468\} & 0.5
\end{array}\right) \text {, }
$$

and its consensus index, denoted as $C I_{H_{1}^{\prime}}=0.1110$.

\subsection{Consensus improving for multiple P-HFPRs}

Based on Algorithms 1 and 2, we now develop Algorithm 3 to improve consensus degrees of multiple H-HFPRs. The symbols in Algorithm 3 are the same as mentioned before.

Algorithm 3. Consensus improving for multiple P-HFPRs

Input: $H_{k}=\left(h_{i j k}\left(p_{i j k}\right)\right)_{n \times n}, \lambda_{k}, k=1,2, \ldots, m, \alpha$.

Step 1 : Based on $H_{k}$ and $\lambda_{k}$, we calculate the group FPR $\tilde{R}=\left(\tilde{r}_{i j}\right)_{n \times n}$ by Eqs. and .

Step 2: According to Algorithm 1, we can obtain $C_{H_{k}}$ with $H_{k}$ and $\tilde{R}$.

Step 3: Select the $H_{\tau}$ satisfying $C I_{H_{\tau}}=\max \left\{C I_{H_{k}} \mid k=1,2, \ldots, m\right\}$. If $C I_{H_{\tau}}>C \bar{I}_{H_{\tau}}$, then go to the next step. Otherwise, go to Step 6 .

Step 4: According to Algorithm 2, we can get $H_{\tau}^{\prime}$ with $\tilde{R}=\left(\tilde{r}_{i j}\right)_{n \times n}$ and $H_{\tau}$.

Step 5: Let $H_{\tau}=H_{\tau}^{\prime}$, then go to Step 1 .

Step 6: Let $H_{k}^{\prime}=H_{k}$ and $C I_{H_{k}^{\prime}}=C I_{H_{k}}$, then end. 
Output: The improved P-HFPRs $H_{k}^{\prime}$ that are with the acceptable consensus degrees, and the consensus indices $\mathrm{CI}_{\mathrm{H}_{k}^{\prime}}$ of $\mathrm{H}_{k}^{\prime}$.

Example 3. Let $H_{1}$ and $H_{2}$ in Example 1 be the inputs of Algorithm 3, $\alpha=0.1$ and Eq. (7) be the selected distance measure in the consensus index. Following Algorithm 3, we can get the improved P-HFPRs, denoted as $H_{k}^{\prime \prime}(k=1,2)$, and their consensus indices, denoted as $C I_{H_{k}^{\prime \prime}}(k=1,2)$, as follows:

$$
\begin{aligned}
& H_{1}^{\prime \prime}=\left(\begin{array}{cccc}
0.5 & 0.3061 & \{0.4,0.5,0.5316\} & 0.3679 \\
0.6939 & 0.5 & \{0.5767,0.6435\} & 0.3970 \\
\{0.6,0.5,0.4684\} & \{0.4233,0.3565\} & 0.5 & \{0.4924,0.5404\} \\
0.6321 & 0.6030 & \{0.5076,0.4596\} & 0.5
\end{array}\right) ; \\
& H_{2}^{\prime \prime}=\left(\begin{array}{cccc}
0.5 & 0.6939 & 0.3393 & \{0.5767,0.6435\} \\
0.3061 & 0.5 & 0.3679 & 0.7111 \\
0.6607 & 0.6321 & 0.5 & \{0.2663,0.3116,0.4\} \\
\{0.4233,0.3565\} & 0.2889 & \{0.7337,0.6884,0.6\} & 0.5
\end{array}\right),
\end{aligned}
$$

where $C I_{H_{1}^{\prime \prime}}=0.1169, C I_{H_{2}^{\prime \prime}}=0.1174$. Clearly, the two P-HFPRs are with the acceptable consensus degrees according to the Table 1.

\section{Discussion}

\subsection{Accuracy of Algorithm 1}

As an iterative method, Algorithm 1 is performed by Monte Carlo simulation in the Matlab environment. As discussed by Zhu and Xu (2014), the outcomes accuracy of the iterative method depends on the total number of iterations. To achieve the accuracy $d$ with 95\% confidence degree for each outcome, the required number of iterations is

$$
K=1.96^{2} / 4 d^{2}
$$

where we set $d=0.01$ as the error limit. For Algorithm 1, we perform 10000 iterations in this paper to guarantee at least $95 \%$ confidence degree for the obtained consensus index.

\subsection{Accuracy parameter}

The values of the accuracy parameter $\alpha$ formalized in Eq. (10) affects the outputs of Algorithms 2 and 3. A smaller value of $\alpha$ implies that the more original preferences the output P-HFPRs preserve. However, it simultaneously requires more iterations to obtain the outcomes. Following Example 2, for different values of $\alpha$, we give the values of $C_{H_{1}^{\prime}}$ and the numbers of the iteration $p$ obtained by Algorithm 2 in Table 2 . 
Table 2. The values of $\mathrm{CI}_{\mathrm{H}_{1}^{\prime}}$ and $p$ with different values of $\alpha$

\begin{tabular}{|c|c|c|c|c|c|}
\hline$a$ & 0.01 & 0.05 & 0.1 & 0.2 & 0.5 \\
\hline$C I_{H_{1}^{\prime}}$ & 0.1211 & 0.1198 & 0.1185 & 0.1160 & 0.1007 \\
\hline$p$ & 51 & 10 & 5 & 3 & 1 \\
\hline
\end{tabular}

From Table 2, it is clear that when $\alpha$ decreases, the numbers of iteration increase, and the consensus degree of the output P-HFPR more approximates the consensus threshold 0.1211 . In this case, the output P-HFPR can mostly preserve original preferences.

\subsection{Consistency checking for P-HFPRs}

Following the approach we developed in this paper to calculate the consensus index, we can similarly develop a consistency index for P-HFPRs. Given a P-HFPR $H=\left(h_{i j}\left(p_{i j}\right)\right)_{n \times n}$, we stochastically sample a FPR, denoted by $H^{l}=\left(h_{i j}^{l}\right)_{n \times n}$, from $H$, where $h_{i j}^{l} \in h_{i j}, i, j=1,2, \ldots, n$. According to Eq., we first get the expected P-HFPR of $H$, denoted by $\bar{H}=\left(\bar{h}_{i j}\right)_{n \times n}$. By Theorem 1 formalized in Xia and Xu (2011b), we then transform $\bar{H}$ into a consistent FPR, denoted by $\tilde{R}^{\prime}=\left(\tilde{r}_{i j}^{\prime}\right)_{n \times n}$. By Eq.(6) or Eq.(7), we calculate the distance between $H^{l}$ and $\tilde{R}^{\prime}$, denoted as $C I_{H^{l}}=d\left(H^{l}, \tilde{R}^{\prime}\right)$. Next, let $H$ be a preference space, then the consistency index that measures the consistency degree of $H$ can be defined as follows:

$$
E\left(d\left(H^{l}, \tilde{R}^{\prime}\right)\right)_{H}=\left(\prod_{i, j=1}^{n} \frac{1}{\left|h_{i j}\right|}\right) \sum_{H} d\left(H^{l}, \tilde{R}^{\prime}\right),
$$

which can be denoted as $C I I_{H}=E\left(d\left(H^{l}, \tilde{R}^{\prime}\right)\right)_{H}$ in brief.

Since the algorithm that calculates the consistency degree of $H$ is similar to Algorithm 1 , we term it Algorithm 4 and omit the detailed derivation for clarity. To compare the consistency index of HFPRs given by Zhu et al. (2014), we give a numerical example as follows:

Example 4. We take a HFPR from Zhu et al. (2014), which is also the P-HFPR $\mathrm{H}_{2}$ in Example 1:

$$
H_{2}=\left(\begin{array}{cccc}
0.5 & 0.8 & 0.3 & \{0.6,0.7\} \\
0.2 & 0.5 & 0.3 & 0.8 \\
0.7 & 0.7 & 0.5 & \{0.2,0.3,0.4\} \\
\{0.4,0.3\} & 0.2 & \{0.8,0.7,0.6\} & 0.5
\end{array}\right) .
$$

Using Algorithm 4 to deal with $\mathrm{H}_{2}$, we can get the value of the consistency degree of $\mathrm{H}_{2}$, denoted as $\mathrm{CII}_{\mathrm{H}_{2}}=0.1865$, against 0.1781 given by Zhu et al. (2014). Zhu et al. (2014)'s method requires three steps to obtain the consistency index, that are estimating an optimized parameter, normalization, and solving an optimization model. However, we can follow Algorithm 4 as an easier approach to get the consistency index with an approximate result. 


\subsection{An example}

The energy channels are significant for a country in transporting power, oil and gas etc. Five experts are invited to estimate the strategic positions of five energy channels. Let $x_{i}$ $(j=1,2, \ldots, 5)$ indicate the five energy channels, respectively, and $H_{i}(k=3,4, \ldots, 7)$ be the P-HFPRs constructed by the preferences provided by the five experts, respectively, denoted as follows:

$$
\begin{aligned}
& H_{3}=\left(\begin{array}{ccccc}
0.5 & 0.3 & \{0.6(0.4), 0.7(0.6)\} & 0.4 & 0.6 \\
0.7 & 0.5 & \{0.4,0.5\} & 0.3 & 0.2 \\
\{0.4(0.4), 0.3(0.6)\} & \{0.6,0.5\} & 0.5 & 0.7 & 0.5 \\
0.6 & 0.7 & 0.3 & 0.5 & 0.2 \\
0.4 & 0.8 & 0.5 & 0.8 & 0.5
\end{array}\right) \\
& H_{4}=\left(\begin{array}{lllll}
0.5 & 0.4 & 0.6 & 0.3 & 0.7 \\
0.6 & 0.5 & 0.6 & 0.4 & 0.4 \\
0.4 & 0.4 & 0.5 & 0.5 & 0.6 \\
0.7 & 0.6 & 0.5 & 0.5 & 0.3 \\
0.3 & 0.6 & 0.4 & 0.7 & 0.5
\end{array}\right) \\
& H_{5}=\left(\begin{array}{ccccc}
0.5 & \{0.2(0.4), 0.3(0.6)\} & 0.6 & 0.3 & 0.6 \\
\{0.8(0.4), 0.7(0.6)\} & 0.5 & 0.6 & 0.4 & 0.3 \\
0.4 & 0.4 & 0.5 & 0.7 & \{0.3,0.4\} \\
0.7 & 0.6 & 0.3 & 0.5 & \{0.4(0.7), 0.5(0.3)\} \\
0.4 & 0.7 & \{0.7,0.6\} & \{0.6(0.7), 0.5(0.3)\} & 0.5
\end{array}\right), \\
& H_{6}=\left(\begin{array}{ccccc}
0.5 & 0.3 & 0.4 & \{0.6,0.7\} & 0.2 \\
0.7 & 0.5 & 0.2 & \{0.3,0.4\} & 0.6 \\
0.6 & 0.8 & 0.5 & 0.9 & \{0.3,0.4\} \\
\{0.4,0.3\} & \{0.7,0.6\} & 0.1 & 0.5 & 0.6 \\
0.8 & 0.4 & \{0.7,0.6\} & 0.4 & 0.5
\end{array}\right) \\
& H_{7}=\left(\begin{array}{ccc}
0.5 & \{0.3,0.4\} & \{0.6,0.7\} \\
\{0.7,0.6\} & 0.5 & \{0.4,0.5\} \\
\{0.4,0.3\} & \{0.6,0.5\} & 0.5 \\
\{0.6,0.5\} & \{0.7,0.6\} & \{0.3(0.5), 0.2(0.25), 0.1(0.25)\} \\
0.4 & 0.8 & \{0.6(0.25), 0.5(0.25), 0.4(0.5)\}
\end{array}\right.
\end{aligned}
$$




$$
\left.\begin{array}{cc}
\{0.4,0.5\} & 0.6 \\
\{0.3,0.4\} & 0.2 \\
\{0.7(0.5), 0.8(0.25), 0.9(0.25)\} & \{0.4(0.25), 0.5(0.25), 0.6(0.5)\} \\
0.5 & 0.2 \\
0.8 & 0.5
\end{array}\right) .
$$

Let $H_{i}(i=3,4, \ldots, 7)$ be the inputs of Algorithm 3, $\lambda_{i}=0.2(i=3,4, \ldots, 7)$ be the weights of the five P-HFPRs, respectively, $\alpha=0.1$ and Eq. (7) be the selected distance measure in the consensus index. Then according to Algorithm 3, we find that only $\mathrm{H}_{6}$ needs improving to achieve the acceptable consensus degree. For convenience, we still use $H_{6}$ in the following to indicate the improved P-HFPR as follows:

$$
H_{6}=\left(\begin{array}{ccccc}
0.5 & 0.3068 & 0.4613 & \{0.5518,0.6749\} & 0.2985 \\
0.6932 & 0.5 & 0.2759 & \{0.3157,0.3959\} & 0.5095 \\
0.5387 & 0.7241 & 0.5 & 0.8540 & \{0.3281,0.4112\} \\
\{0.4482,0.3521\} & \{0.6843,0.6041\} & 0.1460 & 0.5 & 0.5133 \\
0.7015 & 0.4905 & \{0.6719,0.5888\} & 0.4867 & 0.5
\end{array}\right)
$$

In addition, the obtained consensus indices of $H_{i}(i=3,4, \ldots, 7)$ by Algorithm 3 are denoted by $\mathrm{CI}_{\mathrm{H}_{3}}=0.0704, \mathrm{CI}_{\mathrm{H}_{4}}=0.1066, \mathrm{CI}_{\mathrm{H}_{5}}=0.0775, \mathrm{CI}_{\mathrm{H}_{6}}=0.1362$ and $\mathrm{CI}_{\mathrm{H}_{7}}=0.0827$.

To aggregate the preferences in $H_{i}(i=3,4, \ldots, 7)$, we utilize a hesitant fuzzy averaging (HFA) operator introduced by Xia and Xu (2011a). We aggregate the preferences in each line of $H_{i}(i=3,4, \ldots, 7)$, denoted by a HFE $h_{i j}(i=3,4, \ldots, 7 ; j=1,2, \ldots, 5)$, to indicate the aggregation results of the energy channels $x_{j}$ under $H_{i}$, and then aggregate $h_{i j}$ to get $h_{j}$ that indicates the aggregated preferences of energy channel $x_{j}$. With $h_{j}$ and the score function given by Xia and Xu (2011a), we calculate the scores of the five energy channels, denoted as $s\left(h_{1}\right)=0.4946, s\left(h_{2}\right)=0.4905, s\left(h_{3}\right)=0.5503, s\left(h_{4}\right)=0.4969$ and $s\left(h_{5}\right)=0.6927$, respectively. Since a higher score indicates more importance of an energy channel, it is clear that the third energy channel is the most important channel.

\section{Acknowledgements}

The authors would like to thank the editors and the anonymous referees for their insightful and constructive comments and suggestions that have led to this improved version of the paper. The work was supported in part by the National Natural Science Foundation of China (Nos. 61503210, 71571123, 71771155).

\section{Conclusions}

In this paper, we extend HFSs to P-HFSs. As a new tool in group decision making, P-HFSs can better describe the preferences of DMs without information loss. For the applications of 
P-HFSs in group decision making, we develop P-HFPRs and a consensus index to measure the consensus degrees of P-HFPRs. The consensus index obtained by Monte Carlo simulation is reliable with adequate iterations. For inconsistent P-HFPRs, we give an algorithm to improve their consensus degrees until they are acceptable. More importantly, with the preferences characterized by distributions, we can represent big-data preferences by P-HFEs. Consequently, P-HFSs is a new tool in decision making, and shall play an important role nowadays with the development of social media, IT technology and data science.

\section{References}

Alonso, S.; Herrera-Viedma, E.; Chiclana, F.; Herrera, F. 2010. A web based consensus support system for group decision making problems and incomplete preferences, Information Sciences 180(23): 4477-4495. https://doi.org/10.1016/j.ins.2010.08.005

Diamond, P.; Kloeden, P. E. 1994. Metric spaces of fuzzy sets: theory and applications. World Scientific Publishing Company. https://doi.org/10.1142/2326

Dong, Y.; Zhang, G.; Hong, W.-C.; Xu, Y. 2010. Consensus models for AHP group decision making under row geometric mean prioritization method, Decision Support Systems 49(3): 281-289. https://doi.org/10.1016/j.dss.2010.03.003

Herrera-Viedma, E.; Alonso, S.; Chiclana, F.; Herrera, F. 2007. A consensus model for group decision making with incomplete fuzzy preference relations, Fuzzy Systems 15(5): 863-877. https://doi.org/10.1109/TFUZZ.2006.889952

Kacprzyk, J. 1997. Mu ltistage fuzzy control: a prescriptive approach. John Wiley \& Sons, Inc.

Liao, H.; Xu, Z.; Xia, M. 2014. Multiplicative consistency of hesitant fuzzy preference relation and its application in group decision making, International Journal of Information Technology \& Decision Making 13(01): 47-76. https://doi.org/10.1142/S0219622014500035

Orlovsky, S. 1978. Decision-making with a fuzzy preference relation, Fuzzy sets and systems 1(3): 155167. https://doi.org/10.1016/0165-0114(78)90001-5

Torra, V. 2010. Hesitant fuzzy sets, International Journal of Intelligent Systems 25(1): 529-539. https://doi.org/10.1002/int.20418

Torra, V.; Narukawa, Y. 2009. On hesitant fuzzy sets and decision, in 2009 IEEE International Conference on Fuzzy Systems, 20-24 August 2009, 1378-1382. https://doi.org/10.1109/FUZZY.2009.5276884

Wang, J.; Wang, J.; Zhang, H.; Chen, X. 2016. Multi-criteria group decision-making approach based on 2-tuple linguistic aggregation operators with multi-hesitant fuzzy linguistic information, International Journal of Fuzzy Systems 18(1): 81-97. https://doi.org/10.1007/s40815-015-0050-3

Xia, M.; Xu, Z. 2011a. Hesitant fuzzy information aggregation in decision making, International Journal of Approximate Reasoning 52(3): 395-407. https://doi.org/10.1016/j.ijar.2010.09.002

Xia, M.; Xu, Z. 2011b. On consensus in group decision making based on fuzzy preference relations, in Consensual Processes, 267: 263-287. https://doi.org/10.1007/978-3-642-20533-0_15

$\mathrm{Xu}, \mathrm{Z}$. 2004. Goal programming models for obtaining the priority vector of incomplete fuzzy preference relation, International Journal of Approximate Reasoning 36(3): 261-270. https://doi.org/10.1016/j.ijar.2003.10.011

Xu, Z. S.; Xia, M. M. 2011. Distance and similarity measures for hesitant fuzzy sets, Information Sciences 181(11): 2128-2138. https://doi.org/10.1016/j.ins.2011.01.028

Yu, D.; Zhang, W.; Huang, G. 2016. Dual hesitant fuzzy aggregation operators, Technological and Economic Development of Economy 22(2): 194-209. https://doi.org/10.3846/20294913.2015.1012657 
Zadeh, L. A. 1965. Fuzzy sets, Information and Control 8: 338-353. https://doi.org/10.1016/S0019-9958(65)90241-X

Zhu, B.; Xu, Z. 2013. Regression methods for hesitant fuzzy preference relations, Technological and Economic Development of Economy 19(Supl. 1): S214-S227. https://doi.org/10.3846/20294913.2014.881430

Zhu, B.; Xu, Z. 2014. Stochastic preference analysis in numerical preference relations, European Journal of Operational Research 237(2): 628-633. https://doi.org/10.1016/j.ejor.2014.01.068

Zhu, B.; Xu, Z. 2016. Extended hesitant fuzzy sets, Technological and Economic Development of Economy 22(1): 100-121. https://doi.org/10.3846/20294913.2014.981882

Zhu, B.; Xu, Z.; Xia, M. 2012. Hesitant fuzzy geometric Bonferroni means, Information Sciences 205(1): 72-85. https://doi.org/10.1016/j.ins.2012.01.048

Zhu, B.; Xu, Z.; Xu, J. 2014. Deriving a ranking from hesitant fuzzy preference relations under group decision making, IEEE Transactions on Cybernetics 44(8): 1328-1337.

https://doi.org/10.1109/TCYB.2013.2283021 